\title{
Dynamic Evaluation of Deformable Object Grasping
}

\author{
Peng Song ${ }^{1}$, Juan Antonio Corrales Ramón ${ }^{2}$, and Youcef Mezouar ${ }^{1}$
}

\begin{abstract}
Deformable object grasping has great potential to improve the versatility of robotic manipulation and extend the applications of robotics. It has thus attracted more and more attentions and efforts from robotic community. Nevertheless, the deformation of objects during grasping leads to difficulties to evaluate the grasp configurations and to conduct grasping synthesis. Inspired by the dynamic process of deformation, one dynamic evaluation methodology is proposed to extend the static evaluation methods used for rigid object grasping toward deformable object grasping. The dynamic grasp map matrix is formulated to describe the dynamic grasping status of deformable objects. Three dynamic grasp quality metrics are derived from the dynamic grasp map matrix with a discussion on their physical meanings. The specific steps of dynamic evaluation are proposed based on the dynamic metrics. The physical simulation and the robotic experiment have verified the effectiveness of this method. This work is expected to provide new dynamic perspective and solutions for the deformable object grasping synthesis.
\end{abstract}

Index Terms-Grasping, Force and Tactile Sensing, Dynamic Evaluation, Deformable Object

\section{INTRODUCTION}

$\mathbf{R}$ OBOTIC grasping synthesis consists on determining the optimal grasp configuration for robotic tasks. The foundation of grasping synthesis is the definition of effective quality measures to evaluate grasp candidates. For rigid objects, a bunch of quality measures has been proposed and studied in details [1]. These methods attempt to solve two problems: contact location [2] [3] and hand configuration [4]. This article is devoted to solve the problem about contact location. The common measures to determine contact placement include the algebraic properties of grasp map matrix [5] [6], grasp wrench space [7], task wrench space [8] and other measures based on geometric relations [9]. Two important characteristics of the measures for rigid objects are:

- These measures reflect the capacity of one grasp to transfer contact forces as resultant grasp wrench through contact interfaces in direct or indirect form.

- These measures are static because the corresponding transfer capacity is static for a grasp candidate. Because

Manuscript received: September 9, 2021; Revised December 7, 2021; Accepted January 3, 2022.

This paper was recommended for publication by Editor Hong Liu upon evaluation of the Associate Editor and Reviewers' comments. This work was supported by project SOFTMANBOT, which received funding from the European Union's Horizon 2020 research and innovation programme under grant agreement No.869855. JACR was funded by the Spanish Ministry of Universities through a "Beatriz Galindo" fellowship (Ref.BG20/00143).

${ }^{1}$ First Author and Third Author are with CNRS, Clermont Auvergne INP, Institut Pascal, Université Clermont Auvergne, Clermont-Ferrand, France psongfregmail.com, youcef.mezouaresigma-clermont.fr

${ }^{2}$ Second Author is with Centro Singular de Investigación en Tecnoloxías Intelixentes (CiTIUS), Universidade de Santiago de Compostela, Spain jacr56egmail.com

Digital Object Identifier (DOI): see top of this page. the stiffness of rigid body is large enough to resist deformation who can change this capacity.

However, deformable objects have smaller stiffness and their deformation during grasping can bring instability to the transfer capacity. How to evaluate this dynamic transfer capacity is one problem to be solved in this work. First of all, we need to clarify the type of the studied deformable objects since deformable objects can be of different natures with totally different physical properties, such as rope, paper, clothes, 3D toy pieces, plastic bottle, etc [10]. In this paper, volumetric deformable objects with certain stiffness are considered. For common volumetric deformable objects, grasp stability and deformation are two main concerns in grasp evaluation. The grasp stability represents the resistance of a grasp to external disturbance wrenches. For fragile objects or deformable liquid containers [11] preventing large deformation should be the priority. For non-fragile deformable objects, grasp stability is the main problem, which is the focus of our study. After a survey on the existing literatures about deformable object manipulation, several features can be summarized:

- 2D deformable object handling [12]-[14] is more studied than 3D object.

- More efforts are dedicated to analyzing the influence of local changing contact states [15] [16] than to analyzing the varying global wrench transfer capacity.

- The applications are usually limited to some simple and light-loaded situations [15] [17]. To pick up an eggplant or transport a foam do not challenge much on the grasp quality.

In applications, the grasped object could bear different loads to finish tasks. For example, the grasp only needs to overcome the self-weight of object in transportation but has to resist large wrench in assembly. In this case, the interaction between the fingertips and the object should be adapted accordingly. Certainly one static score cannot carry all the information we need to evaluate one grasp during interaction. How to represent and assess this dynamic grasp stability is still an open question.

In this work, a dynamic evaluation method based on the dynamic grasp map matrix is proposed to convey and analyze the dynamic performance of grasp candidates for 3D deformable objects. This method provides two main contributions:

- A new methodology to evaluate grasps on deformable objects in a dynamic manner, which is helpful to inspire new research line.

- A new guidance for grasp synthesis. The specific steps to find the optimal grasp are given based on the analysis of the proposed dynamic metrics.

In order to present the methodology and techniques of dynamic evaluation on deformable objects grasping, the re- 


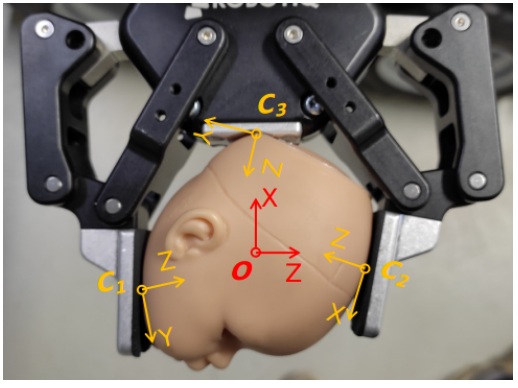

Fig. 1. Contact frames and object frame.

mainder of this work is organized as follows: Section II introduces the mathematical formulations of grasp map matrix and discusses the selection of contact model. Then the dynamic grasp map matrix, three derived dynamic grasp metrics and the specific usage of the metrics in grasp synthesis are presented in Section III. In Section IV, physical simulations demonstrated the procedure of dynamic evaluation method and verified its effect. Section V presents experimental results achieved by one dual arm robotic system to further validate the effectiveness of our dynamic evaluation method. Discussions and concluding remarks are presented in the last section.

\section{Mathematical Formulation OF GRASPing}

\section{A. Grasp Map Matrix}

To describe a grasp, the object frame and the contact frames are set up as shown in Fig. 1. The origin of contact frame is attached on the contact point and the Z-axis of contact frame normally points inward the tangential surface of contact. Each contact point has its own contact frame. The origin of object frame is set on the center of mass of the grasped object. The geometric relationship between the contact frame $C_{i}$ and the object frame $O$ is encapsulated on $g_{o c_{i}}$ :

$$
g_{o c_{i}}=\left(p_{o c_{i}}, R_{o c_{i}}\right) \in S E(3),
$$

where $p_{o c_{i}}$ represents the relative translation between the origins $C_{i}$ and $O . R_{o c_{i}}$ refers to the rotation matrix between contact frame and object frame.

In the contact frame $C_{i}$, the contact model usually has the form as:

$$
F_{c_{i}}=B_{c_{i}} f_{c_{i}} \quad f_{c_{i}} \in F C_{c_{i}},
$$

where $F_{c_{i}}$ represents the contact wrench applied by the fingertip. $f_{c_{i}}$ is a vector representing the contact force magnitude in each dimension. For one certain contact type, the wrench basis $B_{c_{i}}$ is used to define in which dimensions the contact force or torque could be applied. The friction cone $F C_{c_{i}}$ is determined by the friction coefficients between the fingertip and the grasped object [18].

Through the adjoint transformation matrix, the contact wrench $F_{c_{i}}$ can be transferred into the object frame $O$ as $F_{o}$ :

$F_{o}=A d_{g_{o c_{i}}}^{T} F_{c_{i}}=\left[\begin{array}{cc}R_{o c_{i}} & 0 \\ \hat{p}_{o c_{i}} R_{o c_{i}} & R_{o c_{i}}\end{array}\right] B_{c_{i}} f_{c_{i}} \quad f_{c_{i}} \in F C_{c_{i}}$.

One contact map can be defined as:

$$
G_{i}=A d_{g_{o_{i}}^{-1}}^{T} B_{c_{i}} .
$$

In order to derive the general form of grasp map with $m$ contact points, the total wrench in the object frame can be written as the following sum:

$F_{o}=G_{1} f_{c_{1}}+\ldots+G_{m} f_{c_{m}}=\left[\begin{array}{lll}G_{1} & \ldots & G_{m}\end{array}\right]\left[\begin{array}{c}f_{c_{1}} \\ \ldots \\ f_{c_{m}}\end{array}\right]=G f_{c}$.

Combining (4) and (5), the grasp map $G$ can be obtained as:

$$
G=\left[\begin{array}{lll}
A d_{g_{o c_{1}}^{-1}}^{T} B_{c_{1}} & \ldots & A d_{g_{o c_{m}}^{-1}}^{T} B_{c_{m}}
\end{array}\right]
$$

Observing Eq. (6), the grasp map is related to the geometric transformation between contact frames and object frame, and the wrench basis $B_{c_{i}}$ defined by contact model. The geometric transformation is consistent for rigid body but dynamic for deformable objects. If the dynamic geometric transformation can be tracked, we can calculate the grasp map in a dynamic manner and use this dynamic grasp map to analyze the dynamic performance of a grasp.

\section{B. Contact Model}

The grasp quality is usually defined by two general factors. One factor is the wrench transfer capacity formulated by Eq. (6). Another factor is the allowable contact wrench set like friction cone. It defines the range of the transferable contact wrenches.

One contact model usually consists of two components. One component defines the wrench basis $B_{c_{i}}$ which impacts the wrench transfer capacity in Eq. (6). Another component exactly defines the allowable contact wrench set. Since the emphasis of this work is to study the dynamic wrench transfer capacity of a grasp, we should decouple the influences of these two components. The wrench basis $B_{c_{i}}$ should be adjusted according to actual situations. If gripper has small contact with object, the point contact will be used. If the contact area is large, we adopt the wrench basis $B_{c_{i}}$ of the patch contact model. It has the form of $6 \mathrm{D}$ unit matrix, which means 3D force and 3D torque can be transferred through the patch contact interface.

In order to equalize the impact of the allowable contact wrench set, for one object, only the grasp candidates with the same allowable contact wrench set will be considered. For example, the grasp condidates with the similar size of planar contact patch and the same friction coefficients are selected. In this way, we can better reveal the influence of dynamic wrench transfer capacity on actual grasp quality instead of confusing too many factors at the same time. Therefore, in the following contents, the specific allowable contact wrench set is excluded from discussion because it is the same or assumed similar for all grasp candidates of one object.

The different contact models have own wrench basis $B_{c}$. In practice, if $6 \mathrm{D}$ wrenches are generated at the contact, the wrench basis of patch contact model should be used with the form of $6 \mathrm{D}$ unit matrix. If certain contact force or torque along or around one axis is zero or relatively small, the corresponding row of wrench basis can be set as zero. The degraded wrench basis is corresponding to the model of line contact or point contact. For example, the common soft finger 
contact can transfer contact forces along three axes and one contact torque around the inward normal of the tangential contact surface. In total $4 \mathrm{D}$ wrench is transferable in this model. More explanations about wrench basis $B_{c}$ can be found in [18]. For complicated non-planar contacts, an efficient model was proposed to consider the patch contact between the compliant fingertip and rigid objects [19]. This method divides the patch contact into different triangles. The total wrench is the sum of the wrench applied on each triangle. To describe the non-planar friction of patch contact, the 6D limit surface models were defined in [20]. For common planar patch contact between fingertip and soft object, the 3D friction limit surface [21] is used to form the allowable contact wrench set.

\section{DyNAMIC GRASP METRICS AND DYNAMIC EVALUATION}

\section{A. Dynamic Grasp Metrics}

The dynamic grasp map $G_{d y}(t)$ can be expressed in time domain as:

$$
\begin{gathered}
G_{d y}(t)=\left[\begin{array}{ccc}
A d_{g_{o c_{1}}^{T}}^{T}(t) B_{c_{1}} & \ldots & A d_{g_{o c_{m}}^{-1}}^{T}(t) B_{c_{m}}
\end{array}\right] \\
A d_{g_{o c_{i}}^{T-1}}^{T}(t)=\left[\begin{array}{cc}
R_{o c_{i}}(t) & 0 \\
\hat{p}_{o c_{i}}(t) R_{o c_{i}}(t) & R_{o c_{i}}(t)
\end{array}\right]
\end{gathered}
$$

As $B_{c_{i}}$ is fixed with the adopted contact model, the dynamic property of $G_{d y}(t)$ is determined by the relative position $p_{o c_{i}}(t)$ and the relative rotation $R_{o c_{i}}(t)$ between object frame and each contact frame during grasping. This dynamic grasp map $G_{d y}(t)$ represents the dynamic grasping status on the deformable object.

This map is a matrix whose size depends on the number of fingers and the wrench basis $B_{c_{i}}$. Each element of $G_{d y}(t)$ is defined by Eq. (4). If we use soft contact model, as $A d_{g_{o c_{i}}^{T}}^{T}$ is a $6 \times 6$ matrix and $B_{c_{i}}$ is a $6 \times 4$ matrix, the size of each element of $G_{d y}(t)$ is $6 \times 4$. The element number is equal to the number of fingers. The dynamic grasp map $G_{d y}(t)$ will be a $6 \times 12$ matrix for a 3 -finger gripper.

As one matrix, the dynamic grasp map itself cannot be directly used as grasp quality metric. However, each instance of the dynamic grasp map at a moment can be used to calculate grasp metrics or generate grasp wrench space. Concatenating all these values or states together, dynamic grasp metrics can be achieved to assess grasp quality for the entire grasping process. In order to present the idea and method without much complexity, we propose the dynamic grasp metrics based on the algebraic properties of the dynamic grasp map $G_{d y}(t)$. Other quality metrics can also be integrated in this dynamic evaluation methodology as future research work.

A full-rank grasp map $G$ has 6 singular values which are the positive square roots of the eigenvalues of $G G^{T}$. Each singular value quantifies the wrench transfer ability of one grasp in one direction. A higher singular value can transfer a contact wrench as a larger grasp wrench to stabilize the grasped object. Based on this understanding, the minimum singular value of the dynamic grasp map matrix can be used as the first dynamic grasp quality metric, which is expressed as:

$$
Q_{1}(t)=\sigma_{\min }\left(G_{d y}(t)\right)
$$

When the smallest singular value is approaching to zero, the grasp is close to a singular configuration where the grasp is losing resistance to the external wrench in the corresponding direction [5]. $Q_{1}(t)$ reflects the dynamic minimum transmission gain from contact forces to the grasp wrench in the object frame [6]. Thus an effective grasp should keep a large $Q_{1}(t)$.

The dynamic volume of the ellipsoid in wrench space is defined as the second dynamic grasp metric. If we set the constraint $\left\|f_{c}\right\|=1$ (a unitary sphere), (5) can transfer this sphere into an ellipsoid in wrench space. The volume of this ellipsoid reflects one grasp's global transfer ability from contact forces to grasp wrench [5], which has the form:

$$
Q_{2}(t)=\sqrt{\operatorname{det}\left(G(t) G^{T}(t)\right)}=\sigma_{1}(t) \sigma_{2}(t) \ldots \sigma_{6}(t)
$$

where $\sigma_{i}(t)$ denotes each singular value of the dynamic grasp map. This quality metric considers all the singular values with the same weight and should be maximized to achieve the maximum general wrench tranfer ability. Since it cannot reflect the wrench transfer ability of one grasp in a specific direction, we need to use it with other complementary metrics.

After knowing the minimum wrench transfer ability and the global wrench transfer ability, another metric is expected to reflect the equilibrium of transfer ability in all directions. The dynamic grasp isotropy index [6] is defined for this purpose:

$$
Q_{3}(t)=\frac{\sigma_{\min }(G(t))}{\sigma_{\max }(G(t))}
$$

where $\sigma_{\min }(G(t))$ and $\sigma_{\max }(G(t))$ represent the mimimum and maximum singular values of the dynammic grasp map. The value of this metric is between 0 (worst case, singular grasp) and 1 (optimal case, isotropic grasp).

Using these three dynamic metrics together, we can have the thorough knowledge on the dynamic wrench transfer ability of one grasp during grasping. Analyzing these dynamic values can provide the insight to choose the optimal grasp.

Based on our need, these proposed metrics can also relate to other dynamic variables instead of time. When we consider one 2-finger gripper whose contact forces on two fingertips are usually close, the metrics can be formulated as $Q_{1}(f), Q_{2}(f)$, $Q_{3}(f) . f$ refers to the value of contact force.

\section{B. Stability of Wrench Transfer Ability}

Among the proposed three dynamic grasp metrics, we use $Q_{2}$ to represent the global wrench transfer ability (GWTA) of a grasp. During grasping, $Q_{2}$ may have three directions to go: increase, stay constant and decline. We hope it can increase, but in most cases it will decline. It would be constant if the object were rigid. If it has to decline, we hope it could decline as slowly as possible when contact forces are increasing. We use the term "Stability of Wrench Transfer Ability"(SWTA) to describe the ability of one grasp to keep its GWTA. SWTA can be quantified by the gradient of $Q_{2}$. We guess the current local stiffness of object around fingertips has great influence on SWTA. In order to reveal its linkage to physical world, we derived the analytical solution of $Q_{2}$ using Eq. (9) in the simplified case. Then its gradient with reference to contact force is achieved. 
To reduce the complexity of computation, 2-finger grasps are studied. We set the contact frames as the black frames marked in Fig. $3 b$ and the red object frame is located in the center of mass of object. The $Y$ axis of each frame points outward page. The right frame is termed $C_{1}$ and the left frame is termed $C_{2}$. Using Eq. (4) and Eq. (6), the grasp map matrix of one typical 2-finger grasp can be obtained as:

$$
G_{2 f}=\left[\begin{array}{cccccccc}
-1 & 0 & 0 & 0 & 1 & 0 & 0 & 0 \\
0 & 1 & 0 & 0 & 0 & 1 & 0 & 0 \\
0 & 0 & -1 & 0 & 0 & 0 & 1 & 0 \\
0 & -z_{1} & -y_{1} & 0 & 0 & -z_{2} & y_{2} & 0 \\
-z_{1} & 0 & x_{1} & 0 & z_{2} & 0 & -x_{2} & 0 \\
y_{1} & x_{1} & 0 & -1 & -y_{2} & x_{2} & 0 & 1
\end{array}\right]
$$

where $\left(x_{1}, y_{1}, z_{1}\right)$ and $\left(x_{2}, y_{2}, z_{2}\right)$ refer to the coordinates of $C_{1}$ and $C_{2}$ in object frame, respectively. Then we can compute $Q_{2}$ as:

$$
\begin{aligned}
Q_{2} & =\sqrt{\operatorname{det}\left(G_{2 f} G_{2 f}^{T}\right)} \\
& =2\left(z_{1}-z_{2}\right) \sqrt{\left(x_{1}-x_{2}\right)^{2}+\left(y_{1}-y_{2}\right)^{2}+\left(z_{1}-z_{2}\right)^{2}}
\end{aligned}
$$

Two fingers are opposite and in this specific case $\left(x_{1}, y_{1}\right)$ is equal to $\left(x_{2}, y_{2}\right)$. Thus $Q_{2}$ can be simplified as:

$$
Q_{2}=2\left(z_{1}-z_{2}\right)^{2}
$$

If $Q_{2}$ is changing with contact force $f$, its derivation will be:

$$
d\left(Q_{2}(f)\right) / d f=4\left(z_{1}-z_{2}\right)(d(z 1-z 2) / d f)=4 D / K
$$

where $z 1-z 2$ is termed as $D$ that means the distance between two contacts and $K$ is the current stiffness of object between two fingers. Therefore, for 2-finger grasp, we know a larger stiffness can improve SWTA.

\section{Dynamic Evaluation}

To select the grasp with optimal dynamic wrench transfer ability, three factors need to be considered, including SWTA (defined by gradient of $Q_{2}$ ), GWTA (defined by $Q_{2}$ ) and specific transfer ability (defined by $Q_{1}$ and $Q_{3}$ ). At first, we should pay attention to SWTA. If the wrench transfer ability is not stable, the successive discussion will lose fondation. Also, SWTA is related to the object's stiffness. The foundamental impact of stiffness on grasp quality is also discussed in [11]. Then, thinking from whole to part, we should compare GWTA and further consider the distribution problem of wrench transfer ability $\left(Q_{1}\right.$ and $\left.Q_{3}\right)$.

The specific steps of dynamic evaluation can be described as following:

- Compute the dynamic metrics for all grasp candidates.

- Compare SWTA. Exclude the grasps with worse SWTA.

- Compare GWTA. Among the grasps with similar SWTA, we choose the one with the largest GWTA. If SWTA of one grasp is slightly better but its GWTA is much lower than others, we prefer other candidates.

- Compare other factors. If two grasps with similar SWTA and GWTA, we can further compare $Q_{1}$ and $Q_{3}$.

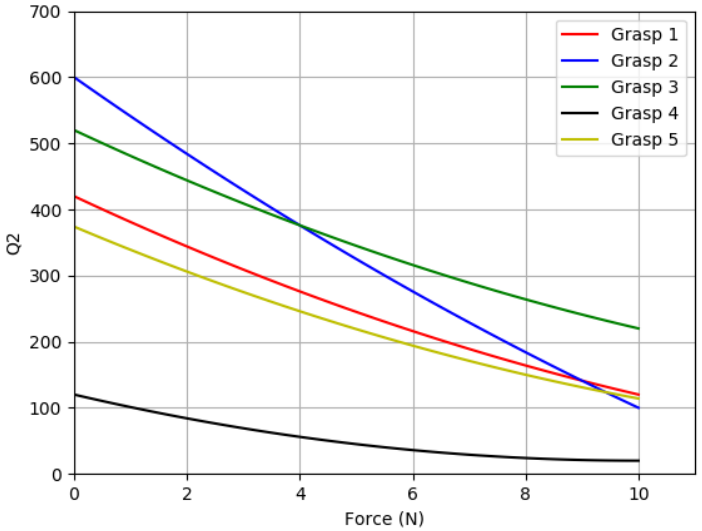

Fig. 2. A set of $Q_{2}$ curves.

For instance, some possible dynamic $Q_{2}$ curves are visualized in Fig. 2. Firstly, Grasp2 is excluded due to its weak SWTA. Then we do not choose Grasp4 because its GWTA is too weak. Finally, Grasp3 is the desired one among these candidates as its good performance in SWTA and GWTA. In the next two sections, this method is applied to analyzing the real dynamic metrics achieved from physical simulations and robotic experiments.

\section{Simulated Dynamic Evaluation}

\section{A. Grasp Quality Prediction}

Using DefGraspSim [22], the contact forces between gripper finger and deformable objects can be fetched to compose the "metrics-force" curves. The grasped object is a deformable brick with the size $6 \mathrm{~cm} * 3 \mathrm{~cm} * 2 \mathrm{~cm}$. We set its Young's Modulus as $2 e^{5}$, Poisson's Ratio as 0.4 , density as $3000 \mathrm{~kg} / \mathrm{m}^{3}$. The franka 2-finger gripper is adopted whose fingers' width is $2 \mathrm{~cm}$. Four grasp candidates are proposed as shown in Fig. 3b. The red object frame is defined at the center of brick. The black contact frames are defined at the center of contact patch. The $Y$ axis points outward page. We denote the configurations indicated by the green, red, yellow and black arrows as Grasp1, Grasp2, Grasp3 and Grasp4, respectively. Grasp1 and Grasp2 are chosen to investigate the impact of stiffness. The comparison among Grasp1, Grasp3 and Grasp4 is helpful to reveal the effect of relative position. The influence of contact area's size is eliminated because the four grasps have the same contact size as $2 \mathrm{~cm} * 2 \mathrm{~cm}$ during grasping. The gripper can downward penetrate the table a bit to grasp the brick since this conflict is set as ignored.

The steps to achieve the dynamic "metrics-force" curves are described as following:

- For one candidate, initialize the gripper's pose as the predefined configuration and maximize its opening.

- Slowly close the fingers. After the contact between finger and brick is detected, we start to record the contact forces and the corresponding positions of fingertips.

- Using the recorded series of fingertip position, we can follow Eq.(1)-(6) to obtain the series of grasp map $G$. 


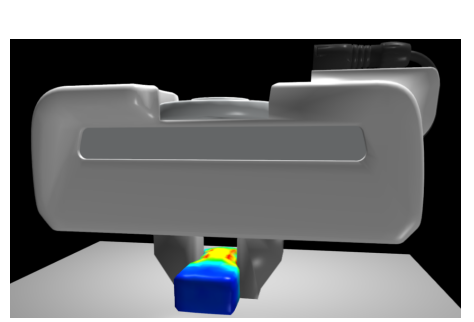

(a)

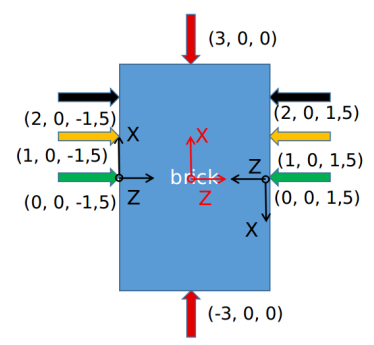

(b)
Fig. 3. Grasp candidates in simulation. (a) Grasp simulation. (b) 4 grasp candidates.

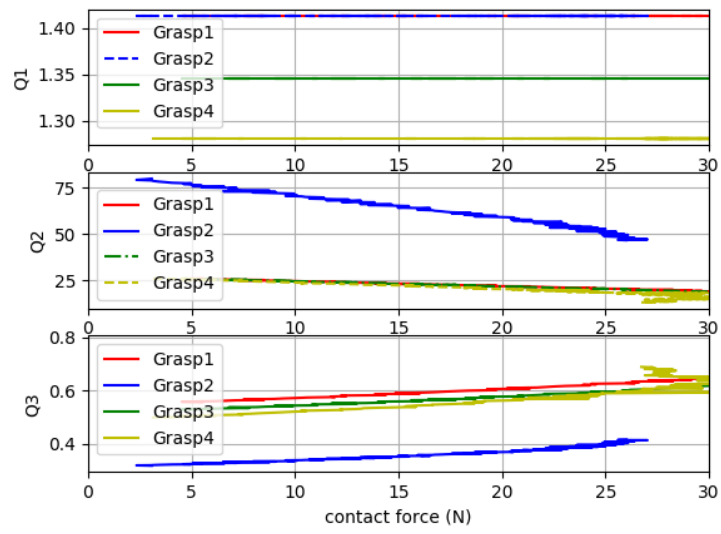

Fig. 4. Dynamic metrics in simulation.

Based on the results of the singular value decomposition on each $G$, we can easily compute the series of metrics by Eq.(8)-(10).

- Visualize these metrics changing with contact force.

For these four grasps, their dynamic metrics are illustrated in Fig. 4. Based on the method in Section III.C, we excluded Grasp2 first because its SWTA is much weaker than other grasps. Since $Q 2$ of Grasp1, 3, 4 are similar, we further compare other factors. In both of $Q 1$ and $Q 3$, Grasp1 is the best and then Grasp3 is better than Grasp4. Therefore, we found "Grasp $1>$ Grasp $3>$ Grasp $4>$ Grasp 2 " according to the analysis of these dynamic curves. This prediction is validated by the stability test in next subsection.

\section{B. Grasp Quality Validation}

Using DefGraspSim [22], we can implement the acceleration tests to verify grasp stability. The test steps can be described as:

- The gripper is initialized to the desired grasp configuration and the desired contact force.

- Select the directions to accelerate.

- The gripper is accelerated slowly along the chosen directions and stops until it loses the grasped brick. The maximum accelerations the gripper can reach before the grasp fails are recorded. The grasp with larger maximum accelerations should be the most stable grasp because it can bear larger inertial forces.

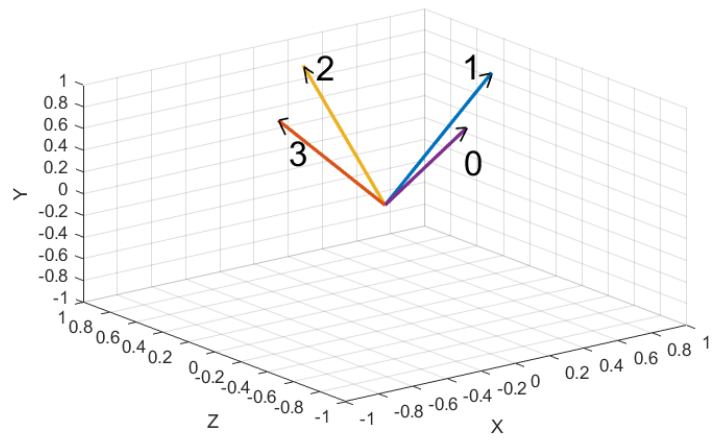

Fig. 5. Directions of acceleration test.

TABLE I

Results of ACCElERAtion Tests

\begin{tabular}{|ccccc|}
\hline Direction & Grasp1 & Grasp2 & Grasp3 & Grasp4 \\
\hline \hline 0 & 83.73 & 52.27 & 71.60 & 56.80 \\
\hline 1 & 62.53 & 61.67 & 62.53 & 69.27 \\
\hline 2 & 87.67 & 53.73 & 68.57 & 57.73 \\
\hline 3 & 64.07 & 62.07 & 90.00 & 62.53 \\
\hline \hline Average & 74.50 & 57.44 & 73.18 & 61.58 \\
\hline
\end{tabular}

The four grasps are tested along the directions " $0,1,2,3$ " defined in Fig. 5 [22]. The desired contact force of each finger is set as $5 N$. The test results are listed in Tab. I. The average value of the maximum accelerations for each grasp verified the prediction from the dynamic evaluation in last subsection.

\section{EXPERIMENTAL DYNAMIC EVALUATION}

This section presents the experimental validation of our method. The "metrics-force" curves are achieved with the help of tactile sensors. By analyzing these curves, the better grasp candidate can be predicted. Then the grasp stability experiments are designed and implemented on a dual arm robotic manipulation platform. We chose a deformable hollow doll head made from rubber as the grasped object, because in the next phase of our project we need to assemble the doll head with doll's other components. In robotic assembly, the doll head will bear a large external wrench thus determining a stable grasp configuration for doll head is crucial. The experimental results proved the effectiveness of the dynamic evaluation to find the more stable grasp disturbed by large external wrenches.

\section{A. Grasp Quality Prediction}

In experiments, the tactile sensors are used to measure contact forces. A pair of tactile sensors (Contactile Sensing Array) is integrated on the 2-finger Robotiq gripper as shown in Fig. 6. Considering the specific design and shape of doll head, two grasp condidates are proposed to assure that the fingers can achieve almost full patch contacts with the object. The relative poses of the two fingers are depicted by the arrows in Fig. 7. It is difficult to find more reasonable candidates who can avoid the area of nose, eyes and ears of toy head. We followed the same procedure to generate the dynamic curves 


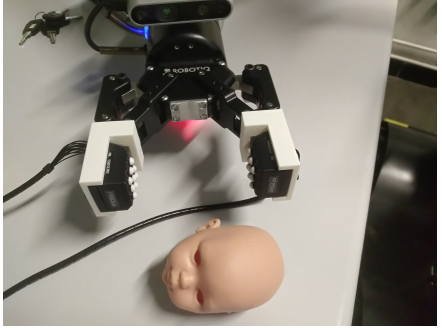

Fig. 6. Integrated tactile sensors.

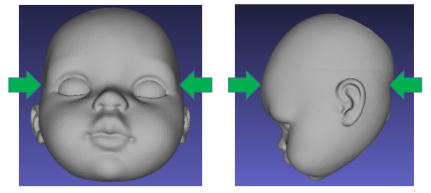

Fig. 7. Two grasp condidates for toy head. The left grasp candidate is named Grasp1 and the right one is named Grasp2.
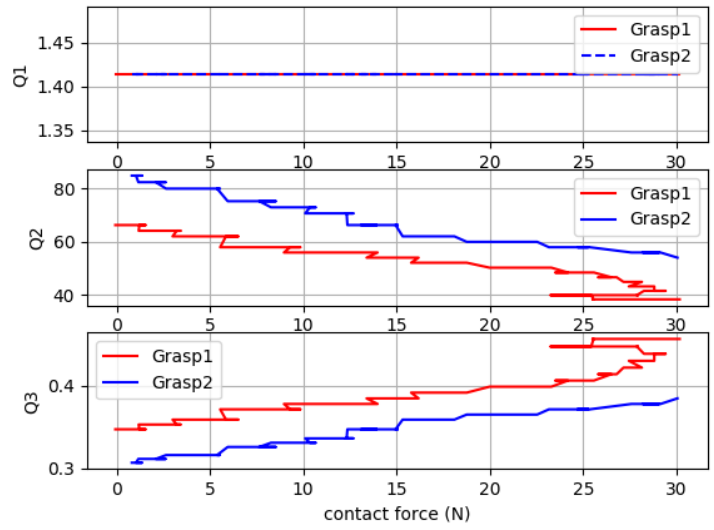

Fig. 8. Dynamic metrics in experiments.

as the steps in simulation. To compute $G$, the wrench basis $B_{c_{i}}$ of patch contact model is adopted because we observed that the fingertips fast achieve the patch contact with toy head and then keep a contact on almost its full rectangle surface.

The achieved dynamic curves for two grasps are illustrated in Fig. 8. It can be observed that Grasp2 has similar gradient and higher values in $Q 2$ compared to Grasp1. It means Grasp2 has similar SWTA and better GWTA. The overlapped curves in $Q 1$ reflect that two grasps have the same minimum transfer ability in one certain direction. Based on Eq. (10), Grasp1's higher value in $Q 3$ implies that the maximum transfer ability of Grasp1 in one direction is weaker than Grasp2. In conclusion we can judge that Grasp2 is more stable than Grasp1.

\section{B. Grasp Quality Validation}

In order to validate the judgement made in last subsection, the "grasping-disturbing-measuring" experiments are designed and implemented. The experimental setup is shown in Fig. 9. The Robotiq 2-finger gripper on the end of the left UR10

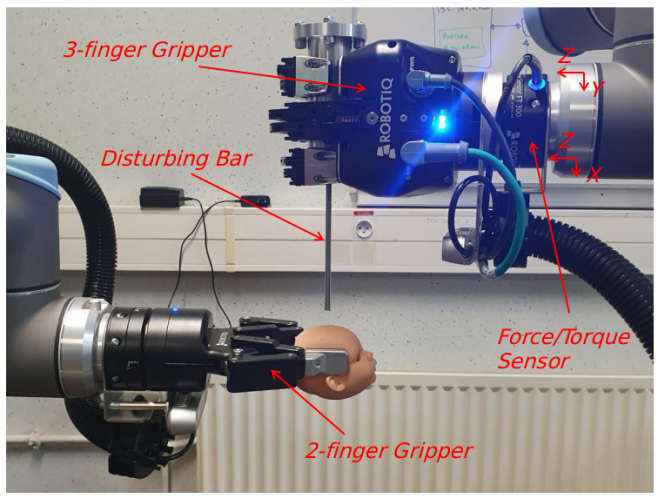

Fig. 9. Experimental setup.

robotic arm is used to grasp the toy head in the configurations defined by Grasp1 and Grasp2. The Robotiq 3-finger gripper on the end of the right UR10 arm is holding the disturbing bar stably. After the 2-finger gripper achieves the desired grasp, the disturbing bar is driven to break the grasp from different directions. During "disturbing", the caused forces and torques will be measured by the force/torque sensor on the wrist joint of the right arm. The frame of force/torque sensor is marked on the right of Fig. 9. The angle between the vertical direction and the $Y$ axis has 45 degrees.

Using the tactile sensors, the map relationship between the gripper separation (the deformation of toy head) and the contact force has been established. For Grasp1, the gripper is controlled to achieve the separation at $48 \mathrm{~mm}$ that corresponds to the contact force around $16 \mathrm{~N}$. For Grasp2, the gripper stops at the opening of $57 \mathrm{~mm}$ which also maps to the contact force around $16 \mathrm{~N}$. Before the start of "disturbing", the grasp will be initialized to these states.

The right arm is controlled to adjust the posture of the disturbing bar. Three groups of experiments are conducted with the initial postures of the bar shown in Fig. 10. In each group, the initial posture, the moving direction, speed and displacement of the disturbing bar are predefined as same with reference to the 2 -finger gripper. The only variation is that we rotate the toy head to switch between Grasp1 and Grasp2. The disturbing bar is driven to move toward the toy head until the grasp ends in failure.

The force/troque sensor on the right arm recorded the interaction during the disturbing process. The measured actual wrenches in these three groups of experiments are visualized in Fig. 11. In Group1 and Group2, the applied external forces/torques on Grasp2 are obviously larger than Grasp1. Grasp2 can resist a larger external wrench until getting invalid, which means Grasp2 can better stabilize the toy head. For Group3, the measured forces on Grasp2 is much larger than Grasp1. However, the measured torques for Grasp1 have a different pattern compared to Grasp2. In fact, this phenomenon is caused by the final status of the toy head stucked between the disturbing bar and the palm of gripper, which is depicted in Fig. 12. In general, the results of these three groups of experiments validated that Grasp2 is more stable than Grasp1, which agrees with the prediction from the dynamic grasp 


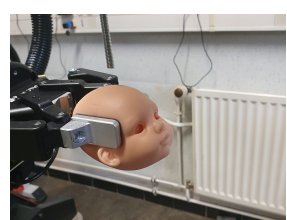

(a)

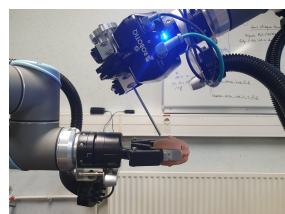

(d)

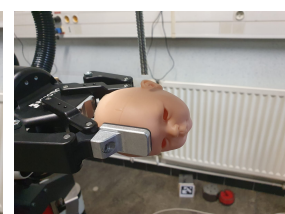

(b)

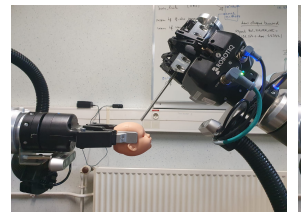

(e)

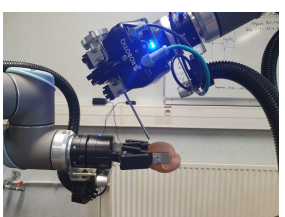

(c)

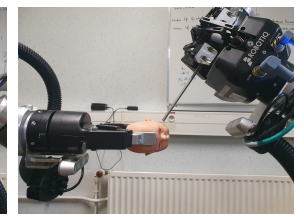

(f)
Fig. 10. Initialization of experiments. (a) Group1-Grasp1. (b) Group1-Grasp2. (c) Group2-Grasp1. (d) Group2-Grasp2. (e) Group3-Grasp1. (f) Group3Grasp2.

quality curves. The effectiveness of the dynamic synthesis method is thus verified.

\section{CONCLUSION}

A dynamic evaluation methodology on deformable object grasping has been proposed, demonstrated and validated to boost the grasping synthesis from static level toward dynamic level. How to exploit the interaction data between gripper and deformable object to guide grasping synthesis is presented in details.

The dynamic grasp map is proposed to describe the varying grasp configuration during the deformation of object. Three dynamic grasp metrics are formulated to quantify the changing wrench transfer ability of a grasp. The linkage between dynamic metrics and physical properties is explained. The analysis method on the dynamic metric curves is provided. These dynamic metrics and the evaluation method are applied in both of physical simulations and robotic experiments. The achieved results proved the effectiveness of the dynamic evaluation in finding the grasp with optimal stability.

This dynamic method brings the possiblity to analyse SWTA of grasp candidates, which is the crucial criterion to find the optimal grasp in consideration of the deformation property of objects. The static methods for rigid objects cannot reflect this factor and cannot be directly applied for deformable objects. For example, when one candidate has high GWTA but low SWTA, it will be excluded by the dynamic method but selected by the static methods. In practice, the low SWTA will lead to the worse grasp stability.

The methodology of dynamic evaluation inspires a new way to analyze, compare and synthesize the grasps for deformable objects. Other grasp quality metrics, algorithms and applications could be filled in this framework to adapt to the requirements defined by the context of handling deformable objects.

\section{ACKNOWLEDGMENT}

This work is supported by a funding from the European Union's Horizon 2020 research and innovation programme under grant agreement No.869855. JACR was funded by the Spanish Ministry of Universities through a "Beatriz Galindo" fellowship (Ref.BG20/00143).

\section{REFERENCES}

[1] M. A. Roa and R. Suárez, "Grasp quality measures: review and performance," Autonomous robots, vol. 38, pp. 65-88, Jan. 2015.

[2] A. Bicchi, "On the closure properties of robotic grasping," International Journal of Robotics Research, vol. 14, pp. 319-344, Apr. 1995.

[3] E. Rimon and J. Burdick, "On force and form closure for multiple finger grasps," in Proc. IEEE International Conference on Robotics and Automation (ICRA'96), Minneapolis, MN, USA, Apr. 1996, pp. 17951800 .

[4] K. Shimoga, "Robot grasp synthesis algorithms: A survey," International Journal of Robotics Research, vol. 15, pp. 230-266, Mar. 1996.

[5] Z. Li and S. S. Sastry, "Task-oriented optimal grasping by multifingered robot hands," IEEE J. Robot. Autom., vol. 4, no. 1, pp. 32-44, Feb. 1988.

[6] B.-H. Kim, S.-R. Oh, B.-J. Yi, and I. H. Suh, "Optimal grasping based on non-dimensionalized performance indices," in Proc. IEEE/RSJ International Conference on Intelligent Robots and Systems (IROS'01), Maui, HI, USA, Oct. 2001, pp. 949-956.

[7] C. Borst, M. Fischer, and G. Hirzinger, "A fast and robust grasp planner for arbitrary 3d objects," in Proc. IEEE International Conference on Robotics and Automation (ICRA'99), Detroit, MI, USA, May 1999, pp. 1890-1896.

[8] _ - "Grasp planning: How to choose a suitable task wrench space," in Proc. IEEE International Conference on Robotics and Automation (ICRA'04), New Orleans, LA, USA, Apr. 2004, pp. 319-325.

[9] C. Rubert, D. Kappler, A. Morales, S. Schaal, and J. Bohg, "On the relevance of grasp metrics for predicting grasp success," in Proc. IEEE International Conference on Intelligent Robots and Systems (IROS'17), Vancouver, BC, Canada, Sep. 2017, pp. 265-272.

[10] J. Sanchez, J. A. Corrales, B. C. Bouzgarrou, and Y. Mezouar, "Robotic manipulation and sensing of deformable objects in domestic and industrial applications: a survey," Intl. Journal of Robotics Research, vol. 37, no. 7, pp. 688-716, Jun. 2018.

[11] J. Xu, M. Danielczuk, J. Ichnowski, J. Mahler, E. Steinbach, and K. Goldberg, "Minimal work: A grasp quality metric for deformable hollow objects," in Proc. IEEE International Conference on Robotics and Automation (ICRA'20), Paris, France, May 2020, pp. 1546-1552.

[12] K. Gopalakrishnan and K. Goldberg, "D-space and deform closure grasps of deformable parts," International Journal of Robotics Research, vol. 24, no. 11, p. 899-910, Nov. 2005.

[13] Y. B. Jia, F. Guo, and H. Lin, "Grasping deformable planar objects:squeeze, stick/slip analysis, and energy-based optimalities," International Journal of Robotics Research, vol. 33, p. 866-897, May 2014.

[14] I. G. Ramirez-Alpizar, M. Higashimori, M. Kaneko, C. H. D. Tsai, and I. Kao, "Dynamic nonprehensile manipulation for rotating a thin deformable object: An analogy to bipedal gaits," IEEE Transactions on Robotics, vol. 28, p. 607-618, Jun. 2012.

[15] H. Lin, F. Guo, F. Wang, and Y. Jia, "Picking up a soft 3d object by "feeling" the grip," International Journal of Robotics Research, vol. 34, p. 1361-1384, Nov. 2015.

[16] L. Zaidi, J. A. Corrales, B. C. Bouzgarrou, Y.Mezouar, and L. Sabourin, "Model-based strategy for grasping $3 \mathrm{~d}$ deformable objects using a multifingered robotic hand," Robotics and Autonomous Systems, vol. 95, p. 196-206, Sep. 2017.

[17] L. Zaidi, J. A. C. Ramon, L. Sabourin, B. C. Bouzgarrou, and Y. Mezouar, "Grasp planning pipeline for robust manipulation of $3 \mathrm{~d}$ deformable objects with industrial robotic hand + arm systems," Applied Sciences, vol. 10, p. 8736, Dec. 2020.

[18] R. M. Murray, Z. Li, and S. S. Sastry, A Mathematical Introduction to Robotic Manipulation. Boca Raton, FL, USA: CRC Press, 1994.

[19] M. Danielczuk, J. Xu, J. Mahler, M. Matl, N. Chentanez, and K. Goldberg, "Reach: Reducing false negatives in robot grasp planning with a robust efficient area contact hypothesis model," in International Symposium on Robotics Research (ISRR), Hanoi, Vietnam, 2019.

[20] J. Xu, T. Aykut, D. Ma, and E. Steinbach, "6dls: Modeling nonplanar frictional surface contacts for grasping using $6 \mathrm{~d}$ limit surfaces," IEEE Transactions on Robotics, vol. 37, no. 6, pp. 2099-2116, Dec. 2021.

[21] I. Kao, K. M. Lynch, and J. W. Burdick, "Contact modeling and manipulation," in Springer Handbook of Robotics. Springer, 2016, pp. 931-954. 

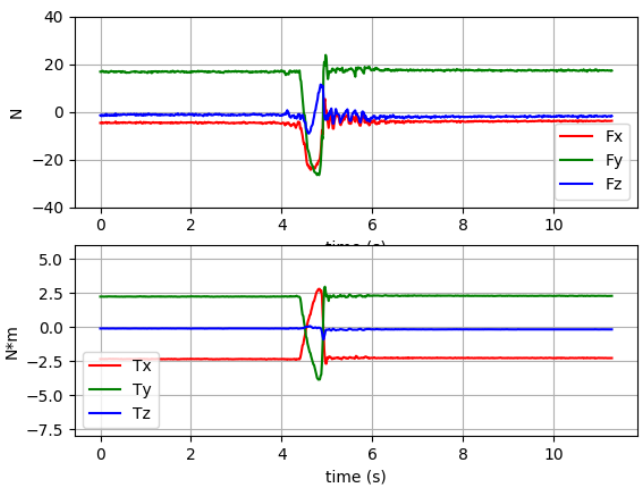

(a) Group1-Grasp1.
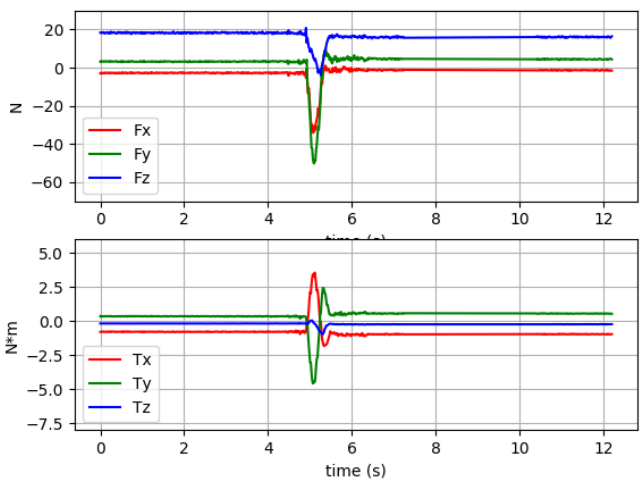

(c) Group2-Grasp1.

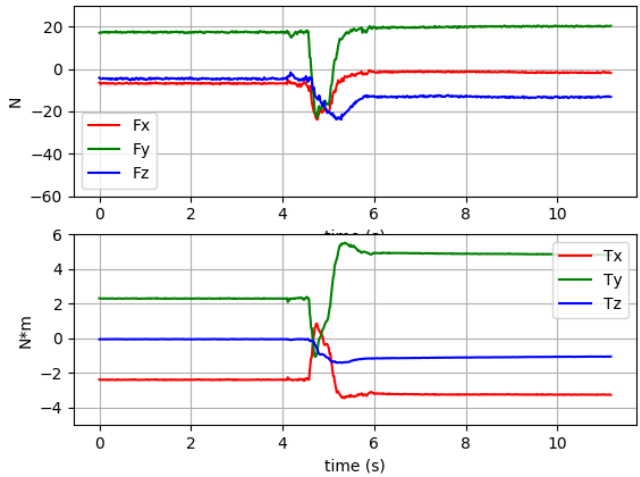

(e) Group3-Grasp1.

Fig. 11. Measured wrenches in experiments.

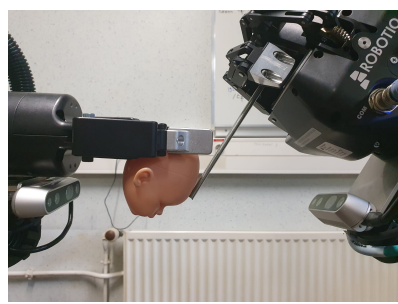

Fig. 12. Stucked toy head.
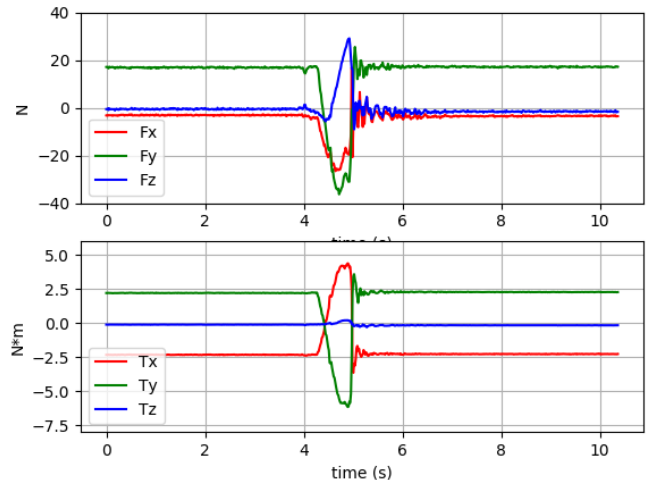

(b) Group1-Grasp2.
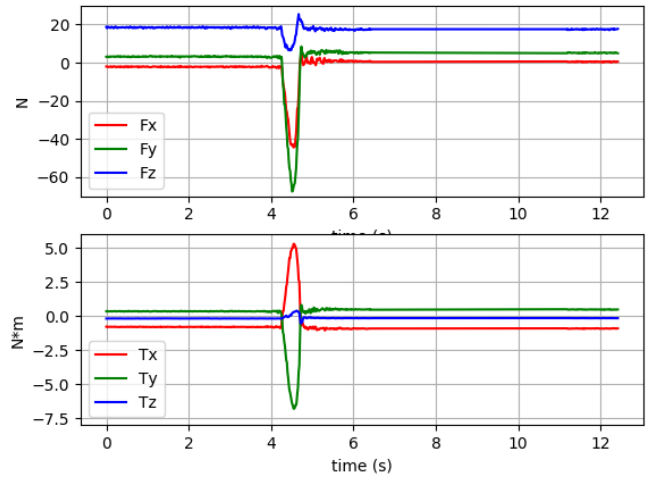

(d) Group2-Grasp2.
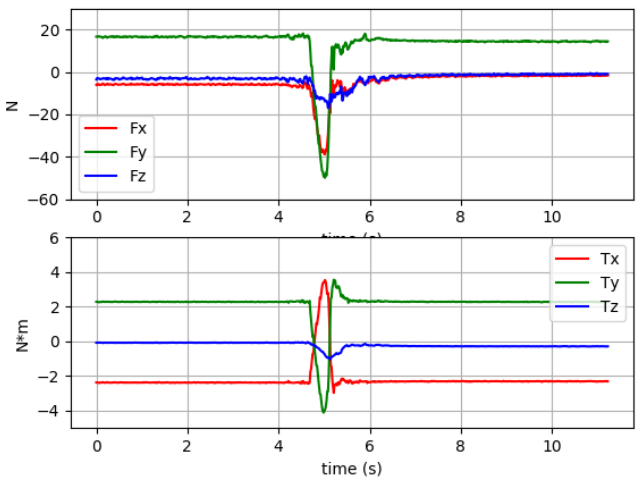

(f) Group3-Grasp2.

[22] I. Huang, Y. Narang, C. Eppner, B. Sundaralingam, M. Macklin, T. Hermans, and D. Fox, "Defgraspsim: Simulation-based grasping of 3d deformable objects," 2021. 